\title{
PRIORIZAÇÃo DA ATRACAÇÃo DE NAVIOS ATRAVÉS DE UM MÉTODO DE APOIO A DECISÃO MULTICRITÉRIO
}

\author{
Nathyelle da Silva Palhano (UEMA) nathyellepalhano@gmail.com \\ Myllena de Jesus Fróz da Silva (UEMA) myllena_jesus@ hotmail.com \\ Yago Everton Inocente da Silva (UEMA) evertonyago31@gmail.com \\ Mônica Frank Marsaro (UEMA) mmarsaro@gmail.com
}

\section{Resumo}

Com o crescimento e o dimensionamento dos países, a atracação dos navios tem se tornado um dos fatores de extrema importância para a determinação da qualidade dos serviços prestados nos portos do mundo. Em se tratando do modal hidroviário ou aquaviário a capacidade de transporte de mercadorias é maior em relação aos outros. Assim sendo, o presente estudo tem como objetivo elaborar um modelo de decisão multicritério para ordenação dos navios para a sua atracação em um determinado porto de uma refinaria. Para tanto, foi utilizado o método PROMETHEE II para a construção do modelo, e o decisor definiu como critérios o demurrage, qualidade do produto a ser descarregado do navio, tempo de operação, dias de estoque do produto na refinaria e ordem de chegada dos navios. Como resultado final teve-se a seguinte ordem dos navios: bauxita da mina II, bauxita da mina I, bauxita da África e navio de carvão. Assim sendo, foi feito uma análise de sensibilidade para a verificação de como os navios se comportavam em relação aos critérios e pode-se perceber que o critério demurrage foi aquele que apresentou maior sensibilidade em relação aos outros.

\section{Palavras-Chaves: PROMETHEE II. PORTO. NAVIO DE BAUXITA}

\section{Introdução}

Ao longo dos anos os países têm-se desenvolvido nos ramos econômicos e tecnológicos, neste contexto o sistema de transporte tem permitido a locomoção de pessoas e mercadorias de um lugar para outro garantindo assim a concretização da eficiência dos serviços. $\mathrm{O}$ autor Ballou (2006, p.150) já afirmava que "um sistema de transportes eficiente contribui para intensificar a competitividade no mercado, aumentar as economias de escala na produção e reduzir os preços dos produtos em geral".

De acordo com Chopra e Meidl (2003), o transporte representa um papel importante para uma cadeia de suprimentos por causa que os produtos raramente são fabricados e consumidos no mesmo local. Segundo Silva (2006), o modal aquaviário é aquele que se diferencia dos outros 
em termos de capacidade, em virtude que o mesmo permite que seja transportado um volume maior de mercadorias com uma variedade de produtos, e Vervloet \& Rosa (2016) afirmam que este modal desempenha um papel fundamental para o comércio internacional, que integrado a outros sistemas de transportes, representa o meio de transporte de grande parte da carga movimentada no mundo.

De acordo com o anuário da Agência Nacional de Transportes Aquaviário (ANAQ, 2019) os portos brasileiros movimentaram 1,005 bilhões de cargas no ano de 2019 e em comparação com o ano de 2018 tem-se uma baixa de 1,80\%, e pode-se afirmar que uns dos problemas dessa diminuição se dar pelos problemas de atracação dos navios que muitos portos enfrentam.

Assim sendo, o objetivo deste artigo é elaboração de um modelo de apoio a decisão para a ordenação de navios para atracarem em um determinado porto de uma refinaria. Diante disso, a possibilidade de se utilizar um método de apoio a decisão multicritério (MCDM - Multicriteria Decision Making) surgiu em decorrência que o mesmo viabiliza á ordenação, classificação ou escolha das alternativas quando se tem mais de um critério que são conflitantes entre si, permitindo assim que o decisor possa analisar os mesmos entre si e priorizar aquele de maior importância (ALMEIDA et al., 2015).

Para tanto, o presente estudo estará dividido em quatro seções: a primeira a introdução, a segunda o referencial teórico sobre problema de atracação de berço, análise multicritério e método PROMETHEE; a terceira é apresentado o estudo de caso e a quarta as considerações finais.

\section{Fundamentação Teórica}

\subsection{Problema de Atracação de Berço}

O problema de atracação de berço (PAB) é uma literatura proposta para o planejamento da ordenação dos navios no porto, em virtude que este problema objetiva na elaboração de uma sequência de atendimento aos navios que estarão aptos a operar no porto (CORDEAU et al., 2005; CERESER,2018). Segundo Bierwirth e Meisel (2010), a função principal de PAB é reduzir o máximo possível o tempo de permanência do navio no porto.

Courdeau et al. (2005) já afirmava que os gestores dos portos constantemente enfrentam duas decisões que são onde e quando os navios devem atracar, e para a tomada dessa decisão é necessário analisar a dimensão espacial e o seu tempo de manuseio do navio. Na dimensão 
espacial são levados em consideração a profundidade da água e a amplitude dos contêiners, no tempo de manuseio do navio é levado em consideração o tempo que o navio leva para descarregar, é uma parte muito importante para o porto, pois é a partir desse tempo que se mede o desempenho do porto.

Segundo Imai et al (2001), nas restrições temporais, que são o tempo de manuseio do navio, podem ser classificados como chegada estatística e chegada dinâmica. Na chegada estatística não é levado em consideração o tempo definido de chegada, pois se considera que todos os navios estão no pátio a espera de serem atracados; enquanto que na chegada dinâmica os navios têm tempo definidos de chegada e não têm permissão para a atracação (BIERWIRTH e MEISEL, 2010; BANOS et al., 2016).

$\mathrm{O}$ PAB é bem recorrente na literatura e nos portos, e o seu estudo é importante para busca de soluções para minimizar o tempo de serviços dos navios e os desvios de tempo de chegada entre a sequência de chegada e a sequência de atracação dos navios (IMAI et al. (2001); IMAI et al. (2008); THEOFANIS et al. (2007)).

\subsection{Análise Multicritério}

O Apoio Multicritério à Decisão (AMD) é uma ferramenta para apoiar à tomada de decisão, hodiernamente utilizado no tratamento de problemas envolvendo escolha, classificação ou priorização de alternativas na presença de critérios múltiplos e conflitantes (GOMES, 2016). Os métodos AMD surgem como solução viável e objetiva, se apresentando como um conjunto de técnicas que auxiliam a análise de tomada de decisão sob influência de um conjunto de critérios (MENDONÇA, 2019). Esses métodos permitem aos agentes decisores estruturarem em um processo decisório, considerando vários aspectos de avaliação, tais como técnicos, socioeconômicos e ambientais, em níveis operacionais e estratégicos pata tomada de decisão (GREGO; FIGEURIA; EHRGOTT, 2016).

A Análise Multicritério tem como objetivo, auxiliar organizações em situações nas quais é necessário identificar prioridades, considerando, ao mesmo tempo, diversos aspectos. (JANNUZZI, P. de M.; WL de MIRANDA; DSG da Silva, 2009), portanto não busca a solução exatamente ótima, mas uma solução consensual negociada em relação aos vários objetivos existentes. A tomada de decisão pode ser definida ainda como um esforço para resolver o dilema dos objetivos conflituosos (ZELENEY, 1982).

Segundo Almeida (2013), um problema de decisão multicritério possui algumas características básicas como, existência de pelo menos duas alternativas de ação, atendimento 
a múltiplos objetivos que muitas vezes conflitantes entre si e a existência de critérios, atributos ou dimensões para avaliar essas alternativas.

Conforme elucidado por Ensslin; Montibeller e Noronha (2001), o processo decisório envolve uma série de etapas dentre elas, a definição clara e objetiva do problema a ser resolvido é crucial, visto que ela norteará todos os demais passos. A modelagem de um problema de decisão multicritério inicia com a definição dos atores que devem participam do processo, que são o decisor, cliente, analista, especialista e terceira parte (ROY, 1996). O decisor é responsável por tomar uma decisão, quando este não possui disponibilidade, o cliente pode assumir esse papel, visto que ele é a figura mais próxima do decisor. O analista é quem detêm o conhecimento acerca do método, por isso será facilitador durante o processo, o especialista é responsável por fornecer informações do problema, já a terceira parte serão afetados pela decisão, porém não participam do processo decisório.

A utilização da análise multicritério para resolução de problemas apresenta como vantagem o fato de tornar possível a construção de uma base para o diálogo entre analistas e decisores, que fazem uso de diferentes pontos de vista, provê facilidade na incorporação das incertezas dos dados sobre cada ponto de vista e ainda permite entender cada alternativa como um compromisso entre objetivos em conflito (BOUYSSOU,1986).

Segundo Almeida (2013), os métodos de análise multicritério podem ser classificados como compensatórios e não compensatórios. A compensação questiona se o bom desempenho de um critério pode ser ou não trocadas pelo mau desempenho de outro, nesse caso considera-se a ideia de trade-off entre os critérios.

\subsection{PROMETHEE}

Os métodos da família PROMETHEE (Preference Ranking Method for Enrichment Evaluation) oferecem aos decisores, a possibilidade de compreender conceitos e parâmetros que simplificam o processo de modelagem de preferências (SILVA et al., 2015). Eles objetivam a construção de relações de sobreclassificação de valores em problemas de tomada de decisão (VINCKE, 1992).

Carvalho e Curi (2016) afirmam que as principais características do método PROMETHEE são a sua simplicidade, clareza e estabilidade, onde a noção de critério generalizado é usada na sua construção de uma relação valorada de sobreclassificação. As relações de sobreclassificação são baseadas em comparação par a par entre as alternativas e não realizam uma agregação analítica para estabelecer notas para cada alternativa. Além disso, método com 
essas especificações são classificados em não compensatórios, portanto não permitem tradeoff.

Esse método é realizado em basicamente duas etapas: construção de uma relação de sobreclassificação e exploração desta. Na primeira fase, deve-se determinar o peso para cada critério e em seguida é definido o grau de sobre classificação através da Equação 1:

$$
\pi(a, b)=\sum_{i=1}^{n} p_{i} F_{i}(a, b), \sum_{i=1}^{n} p_{i}=1
$$

Fi $(a, b)$ é uma função de diferença entre as alternativas $[g(a)-g(b)]$, que assume valores entre 0 e 1 , e pode assumir uma das seis formas apresentadas no seguinte quadro:

Quadro 1: Critérios Gerais PROMETHEE

\begin{tabular}{|c|c|c|}
\hline $\begin{array}{l}\text { 1- Critério usual não há parâmetro a ser } \\
\text { definido }\end{array}$ & $\begin{array}{l}g_{\mathrm{f}}(a)-g_{\mathrm{f}}(b)>0 \\
g_{\mathrm{f}}(a)-g_{\mathrm{f}}(b) \leq 0\end{array}$ & $\begin{array}{l}F(a, b)=1 \\
F(a, b)=0\end{array}$ \\
\hline $\begin{array}{l}\text { 2- Quase-critério define-se o parâmetro q } \\
\text { (limite de indiferença) }\end{array}$ & $\begin{array}{l}g_{\mathrm{f}}(a)-g_{\mathrm{f}}(b)>q \\
g_{\mathrm{f}}(a)-g_{\mathrm{f}}(b) \leq q\end{array}$ & $\begin{array}{l}F(a, b)=1 \\
F(a, b)=0\end{array}$ \\
\hline $\begin{array}{l}3 \text { - Limite de preferência define-se o parâmetro } \\
\text { p (limite de preferência) }\end{array}$ & $\begin{array}{l}g_{\mathrm{f}}(a)-g_{\mathrm{f}}(b)>p \\
g_{\mathrm{f}}(a)-g_{\mathrm{f}}(b) \leq p \\
g_{\mathrm{f}}(a)-g_{\mathrm{f}}(b) \leq 0\end{array}$ & $\begin{array}{l}F(a, b)=1 \\
F(a, b)=[g(a)-g(b)] / p \\
F(a, b)=0\end{array}$ \\
\hline $\begin{array}{l}\text { 4- Pseudocritério definem-se os parâmetros q } \\
\text { (limite de indiferença) e p (limite de preferência) }\end{array}$ & $\begin{array}{l}\left|g_{\mathrm{f}}(a)-g_{\mathrm{f}}(b)\right|>p \\
q<\left|g_{\mathrm{f}}(a)-g_{\mathrm{f}}(b)\right| \leq p \\
\left|g_{\mathrm{f}}(a)-g_{\mathrm{f}}(b)\right| \leq q\end{array}$ & $\begin{array}{l}F(a, b)=1 \\
F(a, b)=1 / 2 \\
F(a, b)=0\end{array}$ \\
\hline $\begin{array}{l}5 \text { - Área de indiferença definem-se os } \\
\text { parâmetros q (limite de indiferença) e p (limite } \\
\text { de preferência) }\end{array}$ & $\begin{array}{l}\left|g_{\mathrm{f}}(a)-g_{\mathrm{f}}(b)\right|>p \\
q<\left|g_{\mathrm{f}}(a)-g_{\mathrm{f}}(b)\right| \leq p \\
\left|g_{\mathrm{f}}(a)-g_{\mathrm{f}}(b)\right| \leq q\end{array}$ & $\begin{array}{l}F(a, b)=1 \\
F(a, b)=\left(\left|g_{\mathrm{f}}(a)-g_{\mathrm{f}}(b)\right|-q /(p-q)\right. \\
F(a, b)=0\end{array}$ \\
\hline $\begin{array}{l}\text { 6- Critério Gaussiano } \\
\text { O desvio-padrão deve ser fixado }\end{array}$ & $\begin{array}{l}g_{\mathrm{f}}(a)-g_{\mathrm{f}}(b)>0 \\
g_{\mathrm{f}}(a)-g_{\mathrm{f}}(b) \leq 0\end{array}$ & $\begin{array}{l}\text { A preferência aumenta segundo uma } \\
\text { distribuição normal } \\
F(a, b)=0\end{array}$ \\
\hline
\end{tabular}

Fonte: Almeida (2013)

Na segunda fase ocorre a definição do fluxo de sobreclassificação, que é realizado através do fluxo de sobreclassificação de saída e o de entrada.

De acordo com Brans e Mareschal (2002), um dos maiores obstáculos do uso do PROMETHEE, está na dificuldade do decisor em entender as funções de preferência, o que funciona como uma barreira para o melhor aproveitamento do método.

A família PROMETHEE é composta por várias versões do método, sendo os principais o I e II, o artigo focará na aplicação do PROMETHEE II, visto que os cálculos do PROMETHEE 
II, de acordo com Olson (1996), resultam numa ordem completa das alternativas, diferente do PROMETHEE I, que fornece um ranking parcial com base nos fluxos.

O PROMETHEE II calcula os fluxos positivos e negativos da preferência para cada alternativa, sendo o fluxo positivo relacionado a situação em que uma alternativa está dominando as outras e o fluxo negativo significa quanto uma alternativa é dominada pelas outras. Ele consiste em simplesmente ordenar as ações pelos números de $\Phi($ a), conforme a Equação 2 demonstra:

$$
\Phi(a)=\Phi^{+}(a)-\Phi^{-}(a)
$$

\section{Estudo de caso}

O estudo foi realizado no porto de uma refinaria localizada em São Luís do Maranhão, e buscou a ordem de atracação dos navios no terminal. O porto estudado possui dois berços para operação, sendo o primeiro utilizado para a descarga de granéis líquidos e para o carregamento do produto final da refinaria, e o segundo, utilizado para a descarga de granéis sólidos, especificamente a bauxita e o carvão. Sendo priorizado as atracações do berço II.

A bauxita, é oriunda de dois fornecedores localizados no Pará, o porto possui seis navios cativos para o ciclo de movimentação, carregamento e descarregamento, da bauxita, pois o minério é a principal matéria-prima da refinaria, e precisa ser descarregada diariamente. Porém, por vezes, quando estes fornecedores não conseguem ofertar o volume do minério demandado pela fábrica, a empresa precisa comprar bauxita internacional, especificamente da África, que possui qualidade inferior em relação a bauxita amazônica. O carvão é recebido por meio de importação, e a frequência de recebimento do material é de apenas 1 navio por mês.

\subsection{Desenvolvimento do modelo}

Para a construção do modelo, algumas etapas foram seguidas de acordo com os estudos da área de análise multicritério de apoio a decisão. A figura 1 ilustra as etapas adaptadas pelos autores para o desenvolvimento do estudo.

Figura 1: Fluxograma da construção do modelo 


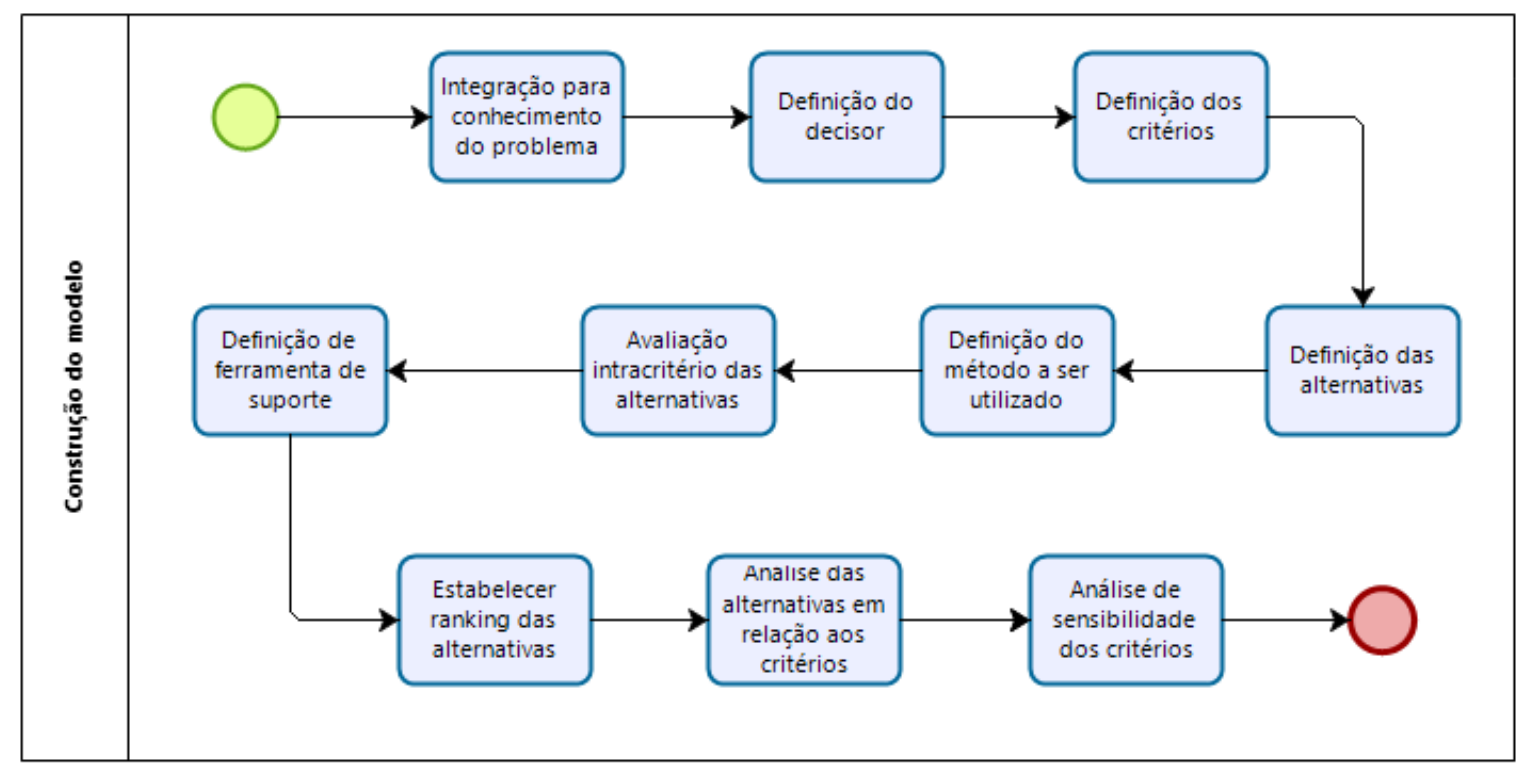

Fonte: Adaptado de Bizagi Modeler (2019)

Inicialmente, foi necessária a realização de um estudo da área, bem como de suas necessidades e restrições, a fim de conhecer a área bem como a problemática de decisão. Dessa forma, foi possível identificar o tomador de decisões do processo que engloba o problema descrito, sendo este o planejador marítimo do porto, uma vez que o mesmo é o responsável por tomar as decisões relacionadas a conflitos de atracação no terminal.

Em seguida, foram identificados os critérios a serem utilizados como parâmetros do modelo, sendo estes:

- Demurrage: taxa que é paga pela sobrestadia do navio em relação ao acordo contratual para tempo de fundeio e operação dos navios no porto. Se trata do maior custo marítimo da empresa, e é calculado por instante de atraso, a partir de 12 horas após a chegada do navio.

- Qualidade: A bauxita possui três indicadores de qualidade cruciais para a área de estocagem e de operação, sendo estes a sílica reativa, a alumina aproveitada e a umidade. Uma vez que a qualidade da bauxita recebida esteja inferior ao acordado em contrato, se faz necessária a utilização de estratégias para o empilhamento deste material no pátio, de forma que a bauxita de qualidade ruim possa entrar no processo produtivo apenas se diluída em uma bauxita de qualidade superior, podendo gerar custos extras para a organização se não preparada com antecedência. Para o carvão, é considerada apenas a umidade, também para estratégias de empilhamento no pátio, de forma que um carvão muito úmido não entre em contato direto com a produção. 
- Tempo de operação: Representa o tempo em que o navio realiza a operação de descarregamento no porto. Os navios oriundos das minas no Pará, por já fazerem parte do ciclo da empresa, operam em 36 horas, já os navios com bauxita oriunda da África ou navios de carvão, precisam passar 48 horas no berço, uma vez que precisa ser realizada uma vistoria de saída nos navios para a liberação da saída, gerando 12 horas ociosas na operação.

- Dias de estoque: Refere-se aos dias disponíveis de estoque do carvão e da bauxita no momento da chegada dos navios.

- Ordem de chegada: Critério correspondente a ordem de chegada dos navios na área de fundeio.

Para a definição das alternativas do modelo, foi realizada a simulação de uma problemática, de forma que o terminal precisasse definir qual navio iria atracar entre 4 opções, sendo estas: navio de bauxita da mina I, navio de bauxita da mina II, navio de bauxita africana e navio de carvão, considerando que todos os navios chegaram em horários próximos.

Para a definição do método a ser utilizado, foram realizadas entrevistas com o decisor, e observou-se que a operação no berço II não sofre impactos relacionados a fatores chamados como Estado da Natureza, e também foi observado, que não existe compensação entre os critérios citados, sendo de relevância para o decisor apenas a importância relativa destes. Sendo assim, com as informações levantadas, definiu-se o PROMETHEE II, como método ideal para a aplicação do modelo.

Na tabela 1, pode-se observar a avaliação intracritério das alternativas, bem como os pesos dos critérios, característica de minimização ou maximização, tipo de função e os parâmetros.

Tabela 1: Dados para a criação do modelo

Critérios

\begin{tabular}{cccccc}
\cline { 2 - 6 } Alternativas & $\begin{array}{c}\text { Demurrage } \\
\text { (per day) }\end{array}$ & Qualidade & $\begin{array}{c}\text { Tempo de } \\
\text { operação }\end{array}$ & $\begin{array}{c}\text { Dias de } \\
\text { Estoque }\end{array}$ & $\begin{array}{c}\text { Ordem de } \\
\text { Chegada }\end{array}$ \\
\hline Navio de BX M1 & $\$ 30.000,00$ & 5 & $36 \mathrm{~h}$ & 17 & 2 \\
Navio de BX M2 & $\$ 30.000,00$ & 4 & $36 \mathrm{~h}$ & 17 & 4 \\
Navio de BX AFR & $\$-$ & 2 & $48 \mathrm{~h}$ & 11 & 1 \\
Navio de carvão & $\$ 17.000,00$ & 4 & $48 \mathrm{~h}$ & 13 & 3 \\
Função & Quase Critério & Usual & Usual & Usual & Usual \\
Parâmetro & $\mathrm{p}=10$ & - & - & - & - \\
Max/Min & Max & Max & Min & Min & Min \\
Pesos & 0,3 & 0,15 & 0,19 & 0,16 & 0,2 \\
\hline
\end{tabular}


O critério qualidade foi avaliado em uma escala verbal de 5 pontos conforme a seguir: 1 Muito ruim; 2 - Ruim; 3 - Médio; 4 - Bom; 5 - Muito bom. Para o critério demurrage foi utilizada a média de demurrage por dia de cada navio, observar que o custo de demurrage do navio de bauxita africana não é de responsabilidade da empresa, devido a acordos contratuais com o fornecedor.

Para o critério demurrage foi utilizada a característica de maximização, pois entende-se que o navio com maior custo de demurrage precisa atracar primeiro, pois o tempo de espera desta causa maior prejuízo financeiro para a empresa. O outro critério com essa característica é o de qualidade, pois uma vez que a qualidade esteja nos padrões desejados não serão ocasionados custos extras de empilhamento.

O critério tempo de operação é caracterizado como de minimização, pois quanto menor o período em que o navio estiver atracado, menor a fila gerada para os navios posteriores. $\mathrm{O}$ critério dias de estoque também possui essa mesma característica, pois quando menor a quantidade de dias do estoque, maior a urgência da produção em relação a este material. E o ultimo critério, ordem de chegada, também possui característica de minimização, pois pela lógica, o primeiro navio a chegar deve possuir prioridade para a operação.

O software escolhido para a execução do método foi o Visual PROMETHEE, já conhecido e bastante utilizado para análises multicritério no mercado. Na tabela 2 pode-se verificar a apresentação do ranking das alternativas, bem como os fluxos positivos e negativos das mesmas.

Tabela 2: Ranking das alternativas

\begin{tabular}{c|cccc} 
Ranking & Alternativas & $\boldsymbol{\Phi}$ & $\boldsymbol{\Phi}^{+}$ & $\boldsymbol{\Phi}^{-}$ \\
\hline $\mathbf{1}$ & Navio de BX M2 & 0,2867 & 0,51 & 0,2233 \\
$\mathbf{2}$ & Navio de BX M1 & 0,17 & 0,4767 & 0,3067 \\
$\mathbf{3}$ & Navio de BX AFR & $-0,2167$ & 0,36 & 0,5767 \\
$\mathbf{4}$ & Navio de carvão & $-0,24$ & 0,3233 & 0,5633
\end{tabular}

Fonte: Autores (2019)

De acordo com o ranking obtido pelo sistema, recomenda-se que o decisor priorize a atracação do navio de bauxita oriundo da mina 2 em relação aos demais. 
Com o intuito de analisar o comportamento das alternativas em relação aos critérios, foi utilizado o gráfico PROMETHEE Rainbow. No gráfico, os retângulos representam os critérios, podendo-se analisar a performance das alternativas para cada um dos critérios de acordo com a largura de cada retângulo. Sendo assim, verificou-se que o navio de bauxita da mina 1 tem maior contribuição positiva dos critérios, enquanto que o navio de bauxita da mina 2 está na média, mas tem a menor contribuição negativa em relação às outras. Os critérios que não aparecem estão na linha zero, ou seja, não contribuem positivamente ou negativamente para a alternativa.

Figura 2: Gráfico PROMETHEE Rainbow

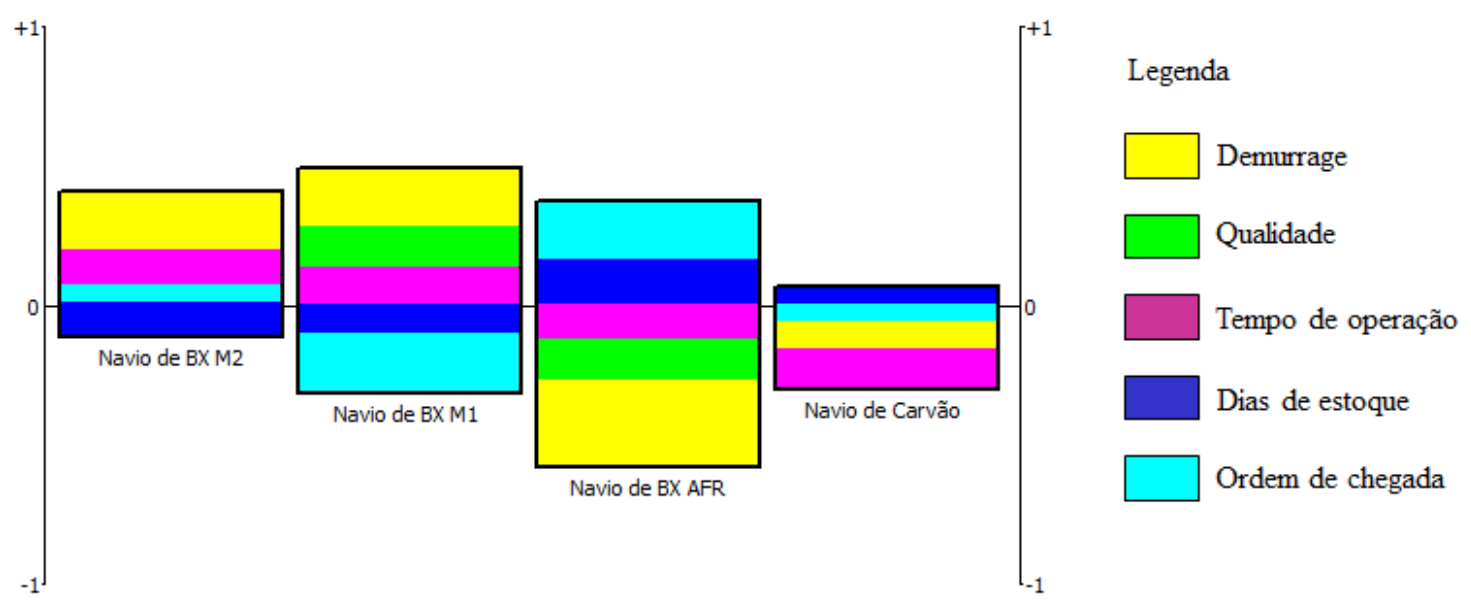

Fonte: Adaptado de Visual Promethee (2019)

No que tange a análise de sensibilidade utilizada para verificar como a alteração do peso dos critérios pode interferir no resultado final, o critério que apresentou maior sensibilidade foi o demurrage, pois caso o peso deste estivesse entre 0,08 e 0,32 , os navios de bauxita das minas 1 e 2 empatariam. O segundo critério mais sensível é o de qualidade, caso este recebesse peso entre 0 e 0,16 , o navio de bauxita oriundo da mina 1 seria o escolhido.

Após a realização das análises, os resultados foram entregues ao decisor e o mesmo considerou a opção sugerida pelo modelo como a mais viável diante do cenário descrito, validando o método como efetivo para resolução de problemas de decisão de atracação de navios no terminal.

\section{Considerações finais}

O processo de decisão para a priorização da atracação de navios nos portos é de grande importância estratégica para a empresa, visto que esta decisão possui alto impacto financeiro e 
na produção da empresa, envolvendo várias particularidades e critérios de escolha, tornando assim, a utilização de um método de apoio a decisão indispensável. Nesse sentido, o método auxiliará o decisor na tomada de decisão para que a escolha seja satisfatória, eficiente e economicamente mais viável.

A utilização do modelo foi possível devido a característica de que é necessário levar em consideração diversos fatores para a escolha da ordem de navios que devem ter prioridade de atracação, sendo assim, para o problema estudado foi aplicado o método PROMETHEE II, com o qual foi possível identificar que opção escolhida deve ser o navio de bauxita oriundo da mina 2, visto que esta alternativa possuiu melhor performance nos critérios definidos quando comparada as demais opções.

\section{REFERÊNCIAS}

ALMEIDA, A. T. Processo de decisão nas organizações: construindo modelos de decisão multicritério. São Paulo: Atlas, 2013.

ALMEIDA, A. T., FERREIRA, R. J. P., CAVALCANTE, C. A. V., 2015. A review of the use of multicriteria and multi-objective models in maintenance. IMA Journal of Management Mathematics, Volume 26, pp. 249$271,2015$.

ANAQ, 2019. Agência Nacional de Transportes Aquaviário. Disponível em:< http://web.antaq.gov.br/ANUARIO/>. Acesso em Jan. 2020.

ARNOLD, J. R. T. Administração de materiais. São Paulo: Atlas, 1999.

BANOS, R. S.; ROSA, R. A.; MAURI, G. R.; RIBEIRO, G. M. Modelo matemático e meta-heurística Simulated Annealing para o problema de alocação de berços com múltiplas carga. Transportes, v. 24, n. 1, p. 51-62, 2016.

BALLOU, R. H. Gerenciamento da cadeia de suprimentos/logística empresarial. São Paulo: Atlas, 2006.

BERTAGLIA, P. R. Logística e gerenciamento da cadeia de abastecimento. 1 e.d. São Paulo: Saraiva, 2003.

BIERWIRTH, C.; MEISEL, F. A survey of berth allocation and quay crane scheduling problems in container terminals. European Journal of Operational Research, v. 202, n. 3, p. 615- 627. DOI: 10.1016/j.ejor.2009.05.031,2010.

BOUYSSOU, D. Some remarks on the notion of compensation in MCDM. European Journal of Operational Research, v. 26, n. 1, p. 150-160, 1986.

BRANS, J. P. \& MARESCHAL, B. PROMÉTHÉE-GAIA: une méthodologie d'aide à la décision en présence de critères multiples. Bruxelas: Éditions de 1l'1université de Bruxelles, 2002.

CARVALHO, J; CURI, W.F. Sistemas de indicadores para gestão de recursos hídricos em municípios: uma abordagem através de métodos multicritério e multidecisor. Revista Brasileira de Gestão e Desenvolvimento Regional, v 12, n. 2, 2016.

CERESER, B.L.H.O problema de alocação de máquinas e berços portuários. Campinas,2018.

CHOPRA, S. e MEINDL, P. Gestão da cadeia de suprimentos: estratégia, planejamento e operações. 1 e.d. São Paulo: Pearson, 2003. DOI: http://dx.doi.org/10.1016/0377- 2217(86)90167-0.

CHOPRA, S. e MEINDL, P. Gestão da cadeia de suprimentos: estratégia, planejamento e operações. 4 e.d. São Paulo: Pearson, 2011

CORDEAU, J.F., LAPORTE, G., LEGATO, P., MOCCIA, L. Models and tabu search heuristics for the berth-allocation problem. Transportation Science, v. 39, n. 4, p. 526- 538. DOI: 10.1287/trsc.1050.0120, 2005. 
ENSSLIN, Leonardo; MONTIBELLER NETO, Gilberto; NORONHA, Sandro Mac Donald. Apoio à Decisão: metodologias para estruturação de problemas e avaliação multicritério de alternativas. Florianópolis: Insular, 2001

GOMES, L.F.A.M. Modelagem do risco no apoio à tomada de decisão. Revista de Ciência, Tecnologia e Inovação, v. 1, n. 1, p. 3-14, 2016.

GRECO, Salvatore; FIGUEIRA, José; EHRGOTT, Matthias. Multiple criteria decision analysis. New York: Springer, 2016.

IMAI, A., NISHIMURA, E., PAPADIMITRIOU, S. Berthing ships at a multi-user container terminal with a limited quay capacity. Transportation Research Part E, v. 44, n, 1, p. 136-151. DOI: 10.1016/j.tre.2007.03.003, 2008.

IMAI, A., NISHIMURA, E., PAPADIMITRIOU, S. The dynamic berth allocation problem for a container port. Transportation Research Part B, v. 35, n. 4, p. 401-417. DOI: 10.1016/S0191-2615(99)00057-0, 2001.

JANNUZZI, P. de M.; WL de MIRANDA; DSG da Silva. Análise multicritério e tomada de decisão em políticas públicas: aspectos metodológicos, aplicativo operacional e aplicações. Informática Pública 11.1: 6987, 2009.

MENDONÇA, Matheus de Melo, et al. Apoio multicritério à decisão para o gerenciamento do sortimento de produtos: um estudo de caso em uma rede de supermercados em Mossoró-RN, 2019.

OLSON, D. L. Decision aids for election problems. In: OLSON, D. L. Decision aids for election problems. New York: SpringerVerlag, 1996.

SILVA, A.C.G.C.; FONTES, C.H.O.; BARBOSA, A.S. Multicriteria evaluation model for organizational performance management applied to the Polo Fruit Exporter of the São Francisco Valley. Computers and Electronics in Agriculture, v. 117, p. 168-176, 2015.

THEOFANIS, S., BOILE, M., GOLIAS, M. An optimization based genetic algorithm heuristic for the berth allocation problem. IEEE Congress on Evolutionary Computation 2007 (CEC 2007). IEEE Computer Society, Washington DC, p. 4439-4445, 2007.

VERVLOET, H. L. L.; ROSA, R .A. Modelo matemático para planejamento da atracação de navios sob o contrato de Charter Party. Transportes, v. 24, n. 1, p. 84-9492, 2016.

VINCKE, P. Multicriteria decision-aid. Bruxelles: John Wiley \& Sons, 1992

ZELENY, M. Multiple criteria decision making. New York: MacGraw-Hill, 1982. 